\title{
Should cool be a design goal?
}

\author{
Benjamin R. Cowan \\ $\mathrm{HCl}$ Centre \\ University of Birmingham \\ b.r.cowan@cs.bham.ac.uk
}

\author{
Katerina Avramides \\ London Knowledge Lab \\ Institute of Education \\ K.Avramides@ioe.ac.uk
}

\author{
Russell Beale \\ $\mathrm{HCl}$ Centre \\ University of Birmingham \\ r.beale@cs.bham.ac.uk
}

\begin{abstract}
Recently $\mathrm{HCl}$ researchers have taken an interest in the concept of cool and how it can be harnessed in the design process. This is due to cool's potential positive impact on adoption and the wider user experience. In this paper we explore the concept of cool and the challenges faced in the study of cool in design. We highlight the lack of rigorous research and causal understanding of cool, the social nature of the concept, and the likely need of heavy marketing strategy to achieve such status as challenges to the setting of cool as a design goal. We propose that more needs to be understood about the behaviour of cool before it can be considered an appropriate design aim.
\end{abstract}

Design. User Experience. Cool Technologies.

\section{INTRODUCTION}

Commercial marketers have long been interested in understanding cool as a concept to aid in creating cool brands and identifying what is the next cool trend. Recently, discussions within $\mathrm{HCl}$ have begun to explore the concept of cool and how we can incorporate the power of cool in design (Holtzblatt 2011; Read et al. 2011). Conceptually, it is thought that if we could design interactions and devices that were cool then this could act as a motivator to adoption and make users' experience more engaging. Given the emerging interest in designing for cool it is timely to critically explore the concept and its potential. Though certainly an appealing idea, we suggest that the nature of coolness undermines the attainability of designing for cool specifically, although potential ways to bestow cool on technology are explored. This paper discusses the origins of cool and what makes something cool. Crucially it also critically explores the concept of cool as a design goal. This paper aims to promote debate in this area and highlight some of the key issues that need further exploration if this concept is to be fully understood and explored.

(C) The Authors. Published by BCS L\&D Ltd. Proceedings of the $27^{\text {th }}$ International BCS Human Computer Interaction Conference

\section{THE ORIGINS OF COOL}

There is general agreement amongst academics that contemporary cool originates in the AfricanAmerican culture of the 1930s (Belk 2006; Nancarrow et al. 2002). It was defined as a nonchalant attitude that served to mask one's emotions and thus protect Africans from the consequences of emotional display. Some trace cool back to slavery when, for example displays of anger would lead to punishment (Belk 2006). However, it is also thought to have served as a defence more recently amongst African-Americans who maintained respect through detachment in the face of prejudice, humiliation and insecure working conditions (Belk 2006; Nancarrow et al. 2002). Cool became associated with the jazz music scene, drug taking and a particular dress code (notably dark clothes and sunglasses), and was defined by membership in a subculture that opposed societal norms. The rise of consumerism in the 1960s led cool to become linked to consumption (Nancarrow et al. 2002). As in the cool culture of the 1930s, today's young people are expressing cool through behaviour, dress code and music. Unlike members of the original cool culture, young people have grown up in a consumer culture and predominantly seek to demonstrate their identity through products (Pountain \& Robins 2000).

\section{DEFINITION AND CHARACTERISTICS OF COOL}

One of the most challenging aspects of researching cool is the difficulty in describing what it truly is as a 
concept. Most people are able to identify something as cool yet when asked to describe the concept people tend to find it hard to articulate. 'Cool' is a word that is universally used to mean 'fun', 'neat', 'great', 'hot', 'fashionable' or 'excellent', and to denote agreement ("that's cool"), rendering much of its use somewhat vague. 'Cool' is also used to mean "the quality of being fashionably attractive or impressive" (Oxford English Dictionary). Attempts to define cool academically have described it as a set of meanings shared by a peer group that is used to highlight group membership (O'Donnell \& Wardlow 2000) and as a social tool to demonstrate autonomy from mainstream society (Warren 2010). It seems from these views that cool acts as a bond within a group structure and an emblem of autonomy for that group from mainstream society. The fear of something cool leaking into the mainstream is the main threat to cool status and is a major concern for style leaders (Nancarrow et al. 2002) presumably due to social judgement concerns and threat to their autonomous display.

Even though a widely adopted definition of cool is somewhat elusive, there is some consensus on its defining characteristics. From the definitions mentioned it seems that two key characteristics of cool are autonomy and exclusivity most notably against the mainstream (Warren 2010), but also other social groups. By definition then cool is a social judgment and requires validation by others within the group. It is, therefore, something that is bestowed by others (Belk 2006; Leland 2005) rather than something that can be consciously constructed. Philosophically, cool therefore seems to be a collectivistic construct. Interestingly, collectivism may be able to co-exist with other perspectives. The sociologist Simmel (1957), writing about fashion, felt that within individuals there was an inherent duality, firstly of collectivism - belonging, or a desire to belong, to a group - and also of individualism - wanting to be an individual within that group. This attitude seems relevant to the discussion of cool - this assumption of mixed philosophical perspectives coexisting within individuals resonates strongly. A further requirement of cool is also 'authenticity' (Nancarrow et al. 2002). The authentic is distinct from the artificial, mass-produced, and commercialised cultural products of the mainstream. However, exclusivity and authenticity on their own do not suffice, they must be accompanied by an attitude of ironic detachment from others (Belk 2006) and a feeling of superiority, defined by some as narcissistic (Nancarrow et al. 2002; Thurlow 2002). Also critical is the manner in which a cool person attains and expresses this authenticity and rebellion against the mainstream it must come about effortlessly (Belk 2006; Moore 2004; Nancarrow et al. 2002). The cool person does not (outwardly) care about what others think, he is silently confident, laid back, and unconcerned.

For the purpose of our discussion we can extract the following defining characteristics; cool is recognised as being cool within a group, it is exclusive and authentic, it is a demonstration of autonomy from mainstream society, it presents an unconcerned attitude and it is expressed effortlessly.

\section{WHAT MAKES SOMETHING COOL?}

The image of cool is expressed through demeanour, gestures, style of walking and talking, choice of music and venues, lifestyle and selective product consumption (Belk 2006; Gladwell 1997; Nancarrow et al. 2002). As we discussed, identity in contemporary culture is often expressed through selective product consumption and so group membership and the perceived coolness that goes with it is principally expressed through consumption of those products. These expressions of cool are thought to be constructed within subgroups as a means of expressing their autonomy from others, principally the mainstream. So something becomes cool when it becomes an expression of the image of cool; that is, the image that fits with the characteristics we discussed earlier, such as exclusivity and authenticity. Group members will adhere to these group norms in an effort to gather cool judgement from peers within this group (O'Donnell \& Wardlow 2000; Thurlow 2002). Those wanting to associate themselves with the group and disassociate themselves from others will also adopt these styles and related products (i.e. others who I feel are cool have this product so by having it too I show a social link with their value set).

A prominent theory of marketers and "coolhunters" on how these expressions of cool emerge is the transference of cool through association. Cool can be transferred to objects and styles through their use or endorsement by someone who is perceived to be cool. Yet this does not explain how a person comes to be perceived as cool in the first place, nor does it fully incorporate the perception of others in cool attainment. The fact is that peoples' desire to acquire cool through adoption of styles and consumption of products might not be realised in others' perception of them (Belk 2006).

Though it is not well understood how group styles are chosen and how cool is transferred to them, what is certain is that cool rarely remains static. Cool styles and products often diffuse across groups, into the mainstream, then fall out of fashion. However its diffusion across groups is not uniform. For example, while loose fitting jeans spread across groups, body piercing and tattoos were not universally adopted amongst teenage groups (O'Donnell \& Wardlow 2000). A bounded 
autonomy perspective offers a compelling explanation on how cool commences, diffuses through groups and changes over time (Warren 2010). It is argued that cool trends start by being a demonstration of rebellion or uniqueness. This trend is picked up dependent on people's individual differences in desire to demonstrate they are against the mainstream. Those high in counterculturalism adopt this unique style or trend quickly. As adoption of the behaviour increases its display of anti-mainstream is diminished, allowing other who have a lower desire for counter-culturalism to adopt the behaviour, and consequently others higher in desire to show their individuality by leaving the trend. This helps explain how a trend can move towards a mass cool and transfers through society (Warren 2010). As a style or product enters the mainstream it will lose its exclusivity and authenticity and, therefore, its coolness, especially for those high in counterculturalism, but also for the mainstream. For example, once a specific product is bought by the mainstream or a bar is frequented by many its coolness dissipates.

\section{CHALLENGES IN CREATING AND DESIGNING COOL}

Although we have discussed what makes something cool and the origins of cool a fundamental question remains; how do we make a design or a technology cool? Commercial marketers have taken three approaches. The first is to imbue a product with cool by having a cool celebrity endorse it. Belk (Belk et al. 2010) uses Michael Jordan's endorsement of Nike basketball shoes to highlight the success of this approach. As discussed previously, the consumer buys the product from the desire to acquire the celebrity's cool, though this desire might not always materialise. This may symbolize a product's access to the mainstream and its acceptability to the masses but also highlight its preservation as a "niche" or "in the know" identity symbol with celebrity endorsement. The endorsement must however be from someone who holds the traits of cool outlined previously.

Another approach has been to keep track of current trends and identify the 'next cool thing', a practice called coolhunting. New trends are set by a minority of style leaders (Nancarrow et al. 2002) who are sought out and their advice used to guide product development. Not all trends will be amenable to diffusion through the general population. Coolhunters must, therefore, spot emerging trends that have the potential to cut across subgroups. Though coolhunting has had some reported success (Gladwell 1997), it lacks any systematic methodology and is largely based on individuals' ability to pick up on potential new trends.

An alternative for creating coolness also lies in developing a brand that is seen to identify itself as more exclusive and separate to the mainstream, an anti-establishment choice. Once that brand is positioned as cool then products created under that brand attain an element of coolness, similar to the idea of cool by association mentioned previously. Examples of this include Apple, once a specialist producer of high-end computers and now a company whose products hold significant market share in all markets they are in. Although they are the object of choice for a significant number of consumers (and thus do not traditionally reflect a niche product) their products still retain a perception of counter culture and individuality, which is fundamental to the brand ethos that positions them outside the mainstream. The use of Apple products is seen as an emblem of buying into this ethos leaving the user symbolizing socially that they identify with this philosophy.

These approaches pose challenges to $\mathrm{HCl}$ practitioners attempting to endow their designs with cool. The first and third requires heavy commercial marketing strategies, years of brand development work and associated costs that are beyond the scope of some research teams or companies. The second requires means to identify trends and capitalise on them that are unreliable at best. Due to the temporal nature of cool serious consideration must be given to the ability to identify what will be cool in the future and how much the technology designed with this express purpose will keep its cool status. Social trends change unexpectedly making them extremely challenging to identify with accuracy. By the time a design is created designers may have missed the zeitgeist leaving the design or product as something behind the times, a laggard rather than the next cool thing. As researchers designing for cool investigate what is cool in their samples it may be too late in identifying what is already brewing on the fringes and what will be the next thing that is cool. Indeed even if cool is reached the bounded autonomy perspective highlights that this cool is finite with much of the social capital gained from having this cool product being tied with how many people adopt the product and its mainstream popularity.

Instead of targeting the image of a technology alone as discussed previously, or trying to spot the next cool thing, could we instead design technology that embeds the defining characteristics of cool and hope for its adoption of cool on this basis? There are several issues to consider with this approach. Firstly, when embedding the traits of coolness in a product it needs to be seen as authentic, effortless and come from a desire to fight the mainstream. It must act as an emblem to show socially that the 
user is against the ever-shifting current mass trend. The fundamental problem with this approach is in the apparent nature of cool as a social evaluation rather than an engrained trait in a technology. The technology says something about its users to others with others making the judgement of what is cool (as we described in previous sections). Building a design that is guaranteed to be seen by others (and perhaps not all others, but a specific subgroup, as this depends on your focus) as cool seems something of a futile pursuit as this is not bestowed by the designers. The fundamental issue here is the unpredictability of cool. Without heavy commercial marketing budgets (as in the case of Apple) how do we create and evaluate the coolness of the technology? If we are aiming for a product that will be adopted by all then how will it gain characteristics such as exclusivity? If we are seeking adoption by a subgroup then how stable will our assessment of cool be? Will approval by other social groups, undermine the perceived cool of our product?

Something that also needs consideration if we are to assume that cool is transferred is the interaction between the design of the interface we wish people to find cool and the technology platform which it is delivered on. They will almost certainly interact in the formation of coolness. Designing for cool may therefore need to weigh up the image of the platform in terms of brand as well as the ability of the platform to function in certain ways that are deemed cool by the users. This links to the point discussed about branding and product coolness by association earlier. Designers also need to be clear about who their target users are. If the aim in creating a cool technology is eventual mainstream popularity this may be at odds with creating or showing that a specific technology is truly cool (a coolness that, as we discussed, is incompatible with mainstream trends). Designers must be aware of the difference between designing for cool and designing for the mainstream, and that cool does not necessarily mean a product becoming hugely popular. For instance bands that are on the fringes for prolonged periods of time take on a certain status for their fans. What is likely key to this is their autonomy from the mainstream. As soon as that band becomes the mainstream its cool reduces, as suggested by the bounded autonomy perspective. A technology at the fringes with a cult following may preserve its cool for a heightened period for its users whereas a mainstream technology should aim to shape itself or be promoted to a more mass market and mass trend based trajectory. As a technology shifts through these cool phases it may in fact affect the view from core consumers of the brand who were the innovators and early adopters. Yet following real cool may not be desirable in wanting to gain market share or indeed if the desire is to reach a mass market. People therefore need to be aware of whom they are designing for and marketing to, as this will affect the approach needed.

What must also not be forgotten when using cool as a design goal is also what purpose it has in the design space. Cool is likely to act as a motivating factor for purchase and for continued interaction with the technology. But this diminishes as the social evaluation of the coolness of this technology reduces. Cool evaluation of the design by users should be aimed for as a gateway for interaction rather than being a goal that overcomes the design of the technology. The technology must function well and serve a purpose for the user and have the "cool" factor to entice users to use it initially. This sort of technology is likely to have a longer life than designing a trend motivated device or interaction method. A more general criticism of using coolness as a design goal lies in a fundamental question of its measurement and how to ensure the coolness of the technology in question. How do you evaluate coolness and when do we know it has been achieved? Furthermore, how stable will our assessment of cool be?

These fundamental questions need to be addressed before we can truly say that cool can be allocated and assessed as a goal and indeed designed for. The difficulty is that such questions are hard to answer with certainty because of our real lack of empirically led understanding of what cool truly is, how it operates as a concept and its impact on product uptake. From our review of the relevant literature, we found the academic research on cool to be scarce, lacking in methodological rigour and convergent viewpoints as well as based more on case study than scientific investigation. Apart from Warren (Warren 2010) very few texts could be found trying to gain scientific insights into the measurement of cool and its diffusion across groups. This fundamentally needs to be addressed before we can be sure of any conclusions we make about the coolness of designs and understand how to target this in the design process.

\section{CONCLUSION}

Given these challenges we caution against setting cool as a formal design goal. While the potential that coolness has shown in driving the adoption of certain products amongst certain populations is appealing, success stories of widespread product adoption on the basis of cool have been supported by heavy commercial marketing rather than being based formally in the design process. A critical conclusion from the discussion presented here is that the coolness of a product mostly does not lie in the product itself but is created by the social association it has. A product acts as an identity signal of a movement away from the mainstream. 
Ripped jeans may have been worn prior to being seen as cool. However, it was only when they acted an identity signal of a group who were autonomous from the mainstream that they acquired cool status (such as in the 90's grunge music scene). Therefore, designing technology that is cool likely relies on its success in tapping into the social image of exclusivity, authenticity and rebellion from the mainstream rather than being made a fundamental design goal in the creation of an interface or technology.

This is not to say that design does not have a role to play in making something achieve cool. It is likely that good $\mathrm{HCl}$ practice acts in setting the ground for cool adoption by products. Whilst coolness is socially bestowed, it is unlikely to be bestowed on things that are extremely complex to use. Products that are easy to use, beautiful, fun and engaging are more likely to be described as cool products. Additionally, for the sake of building a cool brand the design of poorly focused and poorly functioning products is probably not likely to ensure your brand is seen as cool. To set these foundations for cool we must remember core user centred design principles. If deemed socially appropriate, coolness will follow.

\section{ACKNOWLEDGMENTS}

This research was supported by EPSRC Grant EP/I000437/1. The authors would like to thank the anonymous reviewers for their comments that helped improve the manuscript.

\section{REFERENCES}

Belk, R.W. (2006) Cool Shoes, Cool Self. In Eyes just for shoes. Stockholm: Swedish Royal Armoury.

Belk, R.W., Tian, K. \& Paavola, H. (2010) Consuming cool: Behind the unemotional mask. Research in Consumer Behaviour, 12, 183-208.

Gladwell, M. (1997) The Coolhunt. New Yorker, 78-88.

Holtzblatt, K. (2011) What makes things cool? Principles for design. Course Presented at CHI 2011. Vancouver, BC, Canada.

Leland, J. (2005) Hip, the History, New York: Harper Perennial.

Moore, R.L. (2004) We're cool, mom and dad are swell: Basic slang and generational shifts in values. American Speech, 79(1), $59-86$.
Nancarrow, C., Nancarrow, P. \& Page, J. (2002) An analysis of the concept of cool and its marketing implications. Journal of Consumer Research, 1(4), 311-322.

O'Donnell, K.A. \& Wardlow, D.L. (2000) A theory on the origins of coolness. Advances in Consumer Research, 27, 13-18.

Pountain, D. \& Robins, D. (2000) Cool rules: Anatomy of an attitude. New Formations, 39, 7-14.

Read, J. et al. (2011) Understanding and designing cool technologies for teenagers. In Proceedings of the 2011 annual conference extended abstracts on Human factors in computing systems. CHI EA ' 11. New York, NY, USA: ACM, 1567-1572.

Simmel, G. (1957) Fashion. American Journal of Sociology, 62(6), 541-558.

Thurlow, C. (2002) High-schoolers' peer orientation priorities: a snapshot. Journal of Adolescence, 25, 341-349.

Warren, C. (2010) What makes things cool, and why marketers should care. PhD Thesis USA: University of Colorado at Boulder. 